\title{
Polarization fields in nitride nanostructures: ten points to think about
}

\author{
Fabio Bernardini and Vincenzo Fiorentini \\ Istituto Nazionale per la Fisica della Materia and Dipartimento di Fisica, Università di Cagliari, Italy
}

(August 8, 2018)

\begin{abstract}
Macroscopic polarization, both of intrinsic and piezoelectric nature, is unusually strong in III-V nitrides, and the built in electric fields in the layers of nitride-based nanostructures, stemming from polarization changes at heterointerfaces, have a major impact on the properties of single and multiple quantum wells, high mobility transistors, and thin films. The concepts involved in the theory and applications of polarization in nitrides have encountered some resistance in the field. Here we discuss critically ten "propositions" aimed at clarifying the main controversial issues.
\end{abstract}

Keywords: III-V nitrides, spontaneous polarization, piezoelectricity, quantum wells, HEMT

PACS nos.: 73.40.Kp, 77.22.Ej, 73.20.Dx

III-V nitrides are a new frontier of semiconductor physics [1] . One of their basic properties, macroscopic polarization, offers unique opportunities for device design and basic investigations. Polarization manifests itself as built-in electrostatic fields in polarized layers interfaced to each other. These fields affect the characteristics, performance, and response of multilayer nanostructured devices. Here we discuss some theoretical basics and a number of practical implications of polarization fields for nitride nanostructures.

The microscopic foundations of dielectric polarization theory, established in the first half of the present decade [2, 3], identify the zero-field or transverse polarization $\mathbf{P}_{\mathrm{T}}$ of a periodic bulk crystal as the gauge-invariant Berry's phase of its occupied Bloch orbitals, accumulated in an adiabatic transformation of the system from some reference state to its actual state [2, 3]. $\mathbf{P}_{\mathrm{T}}$, which can equivalently be viewed as the integrated polarization current flowing through the crystal during the transformation, has no relation with the charge density of the polarized dielectric. Importantly, $\mathbf{P}_{\mathrm{T}}$ can be calculated accurately from first-principles density-functional calculations [2 7 . The absolute polarization of a material can also be obtained by referencing its polarization to that of a system for which $\mathbf{P}=0$ by symmetry (for wurtzite, this may be the zincblende phase [4]). Although not directly measurable, $\mathbf{P}_{\mathrm{T}}$ is relevant to polarization and fields in finite, e.g. multilayer, systems through classical relations of the sort $\mathbf{P}_{\mathrm{T}}=\overleftrightarrow{\varepsilon}_{0} \mathbf{P}_{\mathrm{L}}$, where $\overleftrightarrow{\varepsilon}_{0}$ is the (measured or computed [5,7]) static dielectric tensor, and $\mathbf{P}_{\mathrm{L}}$ is the dipole moment per unit volume of the finite system, i.e. the termination-dependent longitudinal polarization (also identified with minus the screened field generated by the net polarization charge at the sample surfaces or outer interfaces). It is simple, as discussed below, to generalize such expression to a multilayer systems relevant in technology in terms of the more fundamental $\mathbf{P}_{\mathrm{T}}$ only [8]. In the rest of the paper, any mention of polarization refers to the transverse variant $\mathbf{P}_{\mathrm{T}}$.
In the case of III-V nitrides [9], we deal with the total polarization $\mathbf{P}=\mathbf{P}_{\mathrm{sp}}+\mathbf{P}_{\mathrm{pz}}(\epsilon)$ in a given strain state at zero temperature, in the absence of external fields. A key point is that in wurtzite nitrides the total polarization at zero strain, known as spontaneous, is non-zero and large. The spontaneous polarization has a fixed direction on general grounds $[10]$, and specifically it points

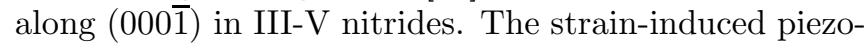
electric term $\mathbf{P}_{\mathrm{pz}}(\epsilon)$ can in principle point in any direction depending on strain. Since nitride multilayers are usually grown along the (0001) axis, $\mathbf{P}_{\mathrm{pz}}(\epsilon)$ actually lies along that axis. Given the results of Ref. [4] and the conventions explained therein, for a layer under tensile strain (lattice constant must expand to fit on substrate, e.g. Al$\mathrm{GaN}$ on $\mathrm{GaN}$ ) the piezoelectric polarization points along $(000 \overline{1})$, whereas for a layer under compressive strain (lattice constant must contract to fit on substrate, say InGaN on $\mathrm{GaN}$ ) it points along the (0001) direction. Similarly to $\mathbf{P}_{\mathrm{sp}}$, the piezoelectric component $\mathbf{P}_{\mathrm{pz}}(\epsilon)$ is also quite unusually large, given the giant piezoelectric constants of III-V nitrides [4, 11.

It is clear that the two components just discussed can sum up or cancel each other out depending on strain and polarity; whether this increases or decreases the field in the layer, depends on the polarization and geometry of the neighboring layers. In fact, the polarization of a material $A$ manifests itself at the interface with a different medium $B$ as a fixed local charge accumulation. If the interface is insulating and gap-states-free (as is generally the case for the isovalent common-anion nitride interfaces), simple relations holds [6,13] between interface charges and bulk transverse polarization. These were verified directly in ab initio calculations on interfaces of binary nitrides [5],6] and of other materials [7]: polarization charge densities of order $10^{13} \mathrm{~cm}^{-2}$, localized in an interface region $\sim 3-4 \AA$ thick are found to be common. As a consequence of these large charge accumulations [14], macroscopic electrostatic fields exist in the interfaced layers, screened by dielectric response [free-carrier screening 
is taken up later on in the paper].

The typical effects of polarization fields are such, as discussed further below, that comparing the predictions of polarization theory with typical experiments is an inherently indirect process, requiring considerable modeling. It is essential that this modeling be done appropriately. We have previously applied [12,13] a sophisticated (and complicated) approach to realistic nanostructures, whereby we solve selfconsistently an empirical tight-binding Schrödinger equation and Poisson's equation. The latter reads

$$
\frac{d}{d z} D=\frac{d}{d z}\left(-\varepsilon \frac{d}{d z} V+P_{T}\right)=e(p-n),
$$

where the position-dependent quantities $D, \varepsilon, V, p, n$, and $P_{T}$ are respectively the displacement field, dielectric constant, potential, hole density, electron density, and total transverse polarization. The effects of composition, polarization, and free carrier screening are thus included in full, and non-equilibrium as well as equilibrium carrier distributions can be studied.

In this paper, instead, we discuss qualitative aspects of polarization effects referring to a simple expression for the the electrostatic field in the $j$-th layer of a periodic but otherwise arbitrary multiple quantum well or superlattice made of layers of materials $k$ of thicknesses $l_{k}$ and dielectric constants $\varepsilon_{k}$ :

$$
\mathbf{E}_{j}=\frac{\sum_{k}\left(\mathbf{P}_{k}-\mathbf{P}_{j}\right) l_{k} / \varepsilon_{k}}{\varepsilon_{j} \sum_{k} l_{k} / \varepsilon_{k}}
$$

with sums running on all layers (including the $j$-th). $\mathbf{P}_{j}$ is the total, spontaneous plus piezoelectric, transverse polarization of the material in layer $j$. This expression, which can be derived [8] under the somewhat restrictive assumptions of periodic boundary conditions and no free carrier screening, shows at a glance that polarization effects couple together all parts of a multilayer structure via polarization differences. Also, simply using Eq. 2 and the values calculated in Ref. [4] for piezoconstants and intrinsic polarization, several often-neglected facts are immediately apparent.

1 - Spontaneous and piezoelectric polarizations are comparable, and neglecting either of the two components leads to inaccuracies. This is best seen in numerical examples; for instance, $\mathrm{Al}_{0.2} \mathrm{Ga}_{0.8} \mathrm{~N}$ assumed to be strained on GaN has a piezoelectric component of $-7.5 \mathrm{mC} / \mathrm{m}^{2}$ and a spontaneous one of $-39.4 \mathrm{mC} / \mathrm{m}^{2}$; an $\mathrm{In}_{0.1} \mathrm{Ga}_{0.9} \mathrm{~N}$ layer strained on $\mathrm{GaN}$ has $+15.6 \mathrm{mC} / \mathrm{m}^{2}$ piezo, and $-29.3 \mathrm{mC} / \mathrm{m}^{2}$ spontaneous. This reflects directly on fields. For a strained $\mathrm{GaN} / \mathrm{In}_{0.1} \mathrm{Ga}_{0.9} \mathrm{~N}$ isolated QW (given the of GaN spontaneous polarization -29 $\mathrm{mC} / \mathrm{m}^{2}$ ) one gets a field of $30 \mathrm{kV} / \mathrm{cm}$ on neglecting the piezo component, while the full value is $-1.63 \mathrm{MV} / \mathrm{cm}$. For an unstrained isolated $\mathrm{Al}_{0.2} \mathrm{Ga}_{0.8} \mathrm{~N} / \mathrm{GaN} \mathrm{QW}$, neglect of spontaneous polarization gives a field of -1.14
$\mathrm{MV} / \mathrm{cm}$ instead of the actual $-1.89 \mathrm{MV} / \mathrm{cm}$. The effect is less dramatic in the $\mathrm{AlGaN} / \mathrm{GaN}$ systems since the piezoconstants of AlN are extremely large so that the piezoelectric term is sizable even at low strain. Of course, besides the errors it causes, neglect of either of the components is conceptually ill-founded.

2 - Only polarization differences at interfaces are relevant. This fact is a consequence of electrostatics via $\nabla \cdot \mathbf{P}=-\sigma$, it is consistent with the tenet of basic polarization theory [2] that only polarization differences are meaningful, and is also borne out by Eq. 2, which is derived under simple electrostatics assumptions. Some workers incorrectly assumed earlier on, that in a generic MQW the field in the active (well) region was given by the polarization/dielectric constant ratio of the well material only. In the case of e.g. a GaN-matched MQW with equally thick layers of $\mathrm{GaN}$ and $\mathrm{Al}_{0.2} \mathrm{Ga}_{0.8} \mathrm{~N}$, this assumption leads to predict a field of $-3.2 \mathrm{MV} / \mathrm{cm}$ in the well, while using the correct procedure the fields are, as expected, equal and opposite in the well and barrier, and $0.74 \mathrm{MV} / \mathrm{cm}$ in modulus.

3 - The geometry of the structure determines directly the fields. This comes about for two reasons. First, the field is determined by the structure geometry via Eq. 22; second, the field-induced potential drop changes with layer thickness. In a GaN-matched $\mathrm{Al}_{0.2} \mathrm{Ga}_{0.8} \mathrm{~N} / \mathrm{GaN}$ system with $30 \AA$ wells and $60 \AA$ barriers the field in the wells is $-1.31 \mathrm{MV} / \mathrm{cm}$ with a potential drop of $0.39 \mathrm{eV}$; if the wells are enlarged to $60 \AA$, the well field is $-0.98 \mathrm{MV} / \mathrm{cm}$, and the potential drop is $-0.59 \mathrm{eV}$. Hence, doubling the thickness of a single layer does not straightforwardly produce a doubled potential drop. This is most relevant since it is just this potential drop that causes the optical shifts visible in PL measurements. Note in passing that the number of barrier/well units and their growth sequence is also relevant.

4 - Piezo vs spontaneous dominance depends on composition. Nanostructures involving AlGaN/GaN will be dominated by spontaneous fields, due to the huge difference in spontaneous polarization between AlN and GaN, and their relatively small lattice mismatch. $\mathrm{GaN} / \mathrm{InGaN}$ nanostructures will be dominated by piezoelectricity, since the spontaneous polarization difference between InN and $\mathrm{GaN}$ is small, whereas their lattice mismatch is large. This becomes obvious considering that, in the Vegard hypotesis, for each $10 \%$ molar fraction change the specific strain is $-1 \%$ for $\mathrm{InN}$ in $\mathrm{GaN}$, and $+0.25 \%$ for AlN in GaN, while $\Delta \mathbf{P}_{\mathrm{sp}}$ is $+0.3 \mathrm{mC} / \mathrm{m}^{2}$ and $-5.2 \mathrm{mC} / \mathrm{m}^{2}$ for the same cases.

The interplay of free carriers (intrinsic, extrinsic, injected, photogenerated, etc.) and polarization fields in nitride multilayers turns out to give rise to a rich variety of new phenomena. A closer look to these issues through accurate simulations and thoughtful experiments has indeed revealed several hot spots: 
5 - Optical transitions are red-shifted, slowed down, and made less intense by the fields. This effect, due to the progressive spatial separation of hole and electrons at the two sides of the QW, was observed by several workers in strained GaN/InGaN [15], and strained as well as unstrained AlGaN/GaN MQWs 16,17]. In calculations, it was shown that at excitation densities typical for PL measurements, the shift in a single QW is perfectly linear with the thickness, and the oscillator strength is suppressed accordingly [12,13,18]. The shifts are most often such that the transition occurs well below the band gap energy. Identifying the magnitude of the polarization via measurement of the shifts requires an accurate modeling, expecially when different well thicknesses are used at the same time in a MQW [17], and also in the small-thickness limit where the field-induced red shift is not linear due to the competing confinement blue shift.

6 - Optical excitation, electrical injection, or doping, lead to partial screening of the fields due to generation of free carriers in the active layers or thereabouts. This was observed in experiment as a blue shift of the transition energy and recovering oscillator strength as a function of excitation power 12, 15. As indicated theoretically [12,13], the blue shift is in fact relative to the field-red-shifted energy, and failure to recognize the red shift as due to polarization has lead to overestimates of the In contents in InGaN layers. Referring to Fig. 1, more points are worth a mention.

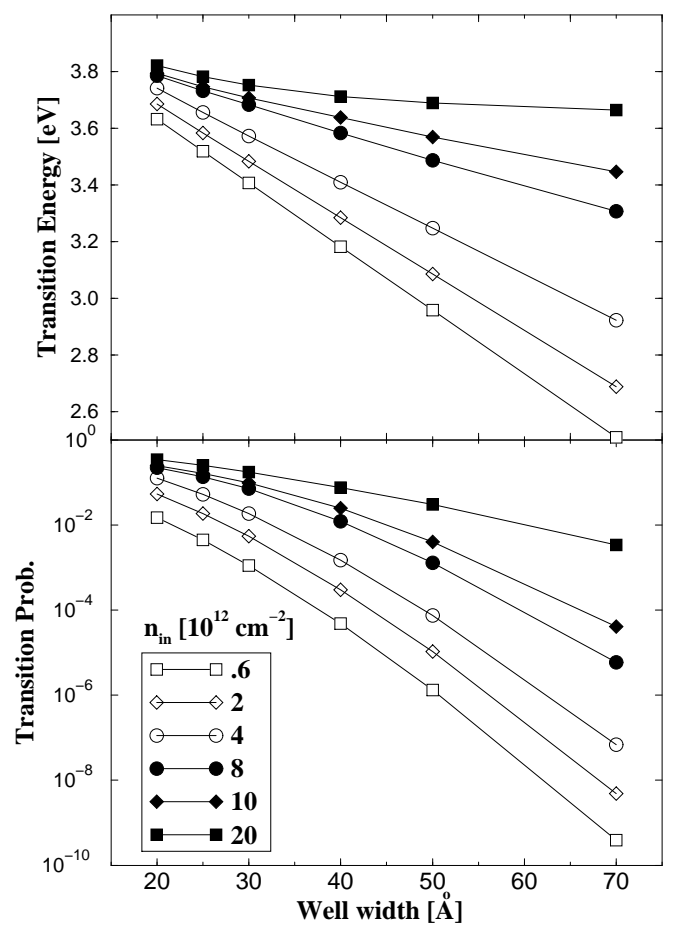

FIG. 1. Transition energies and probabilities of an isolated $\mathrm{Al}_{0.2} \mathrm{Ga}_{0.8} \mathrm{~N} / \mathrm{GaN}$ quantum well as function of well thickness and excitation density (see Ref. [13])
A very substantial excitation is needed to recover appreciable oscillator strengths, and to blue-shift the transition back towards the flat-band value; this finding agrees with thresholds of GaN-based lasers being up to one order of magnitude larger than in GaAs-based devices. Further, the oscillator strength never reaches unity, and the energy never equals the gap plus confinement energy even in the highest power limit. This is because the relevant length scale for free carrier screening of the field is of order 15-20 $\AA$ due to the larger spatial extension of the quantum-confined carriers as compared to the polarization charge. A possible exception are extremely thin wells where the screening length is similar to the well width, and the confinement blue shift competes with the field red shift. Note that while excitation-driven screening dies out as excitation is removed, permanent screening can be and has been achieved by remote doping [13,19]. 7 - The field-induced potential drop can become similar to the gap of the layer material, for typical fields (several $\mathrm{MV} / \mathrm{cm})$ over typical well thickness $(\sim 100$ $\AA$ ). Screening by the intrinsic carriers becomes important in this limit. As could be guessed, simulations show that at non-zero temperature, as a result of free carrier generation at the opposite ends of the sample, the fieldinduced potential drop across a given layer never exceeds the gap, but rather [13] the field obeys $|\mathbf{E}|=E_{\text {gap }} / d$ for thicknesses $d \geq d_{c}=E_{\text {gap }} /|\mathbf{E}|$. Since the relevant $d_{c}$ 's are generally larger than typical QW thicknesses, the gap closure regime is generally not of interest there, but it may be to thick layers (used e.g. to measure alloy properties). On the other hand, cases exist where intrinsic carriers determine the boundary conditions, as in the next point.

8 - Self-doping may occur in "constrained" systems. This was observed experimentally by at least three groups 202 22] and confirmed theoretically 23] in the instance of high mobility transistors. We pick the example of a thick $(\sim 300 \AA)$ AlGaN layer on a very thick ( $1.5 \mu \mathrm{m})$ GaN buffer, contacted at the top. The metal Fermi level, the Schottky contact barriers, and the interface band offset limit the maximum admissible potential drop through the AlGaN layer to about $1 \mathrm{eV}$. The polarization field would instead imply a drop of about 4 $\mathrm{eV}$. For equilibrium to be established, a large part of the polarization charge at the heterointerface must therefore be screened by free carriers, coming in from the GaN layer (not from the contact due to the Schottky barrier) following the strongly attractive polarization-induced potential drop on the GaN side. The GaN layer is typically unintentionally $n$-doped to at least $10^{16} \mathrm{~cm}^{-3}$, hence a fair source of electrons. The end result at equilibrium 2023 is the accumulation of a extremely high-density (up to $10^{13} \mathrm{~cm}^{-2}$ ) two-dimensional electron gas at the $\mathrm{AlGaN} / \mathrm{GaN}$ heterointerface channel (the mechanism is summarized, e.g., in Ref. [13] and discussed in detail 
in Refs. 20], 22], and [23]). The mobility and channel transconductance are increased accordingly [23], also thanks to the absence of ionized impurities in the channel itself. Two groups 20,21 have interpreted their results in terms of piezoelectricity only, while a third [22] has included spontaneous polarization. At the level of charge density CV profiling measurements, the uncertainties are sufficiently large, and the interpretations indirect enough that both ways of proceeding can get acceptable credit; as mentioned in the next point, however, only spontaneous polarization stands the consistency check with the sample polarity.

Two-dimensional hole gases can also be obtained thanks to fields, as demonstrated recently 24. A superlattice is designed so that the field in the doped barriers and well is equal and opposite; this is achieved to a good approximation with a MQW with equal well and barrier thickness ( see Eq. 2). The field enhances, essentially by field ionization, the release of holes from the relatively deep acceptors in the GaN or alloy layer, and funnels the holes to the interface holding negative polarization charge. Holes accumulate at the interface forming a degenerate 2-D gas. Spontaneous polarization was accounted for, and measured charge densities matched well those predicted by modeling.

9 - Polarity plays a key role: since the polarization in each layer has a definite sign dictated by strain and by the spontaneous polarization direction, the combination of polarizations to produce fields (Eq. 2) is determined by the polarity of the structure. This is in turn determined by the substrate- and preparation-dependent growth face $[\mathrm{N}-$ face, i.e. $(000 \overline{1})$ surface up, or rather Ga-face, i.e. (0001) surface up]. Hence control on, and awareness of the polarity issue is also essential, since it is easy to envisage how polarity influences experiments. The measurement of polarization effects through fieldinduced optical shifts in quantum wells is "sign-blind", since the field sign is immaterial to the observed shift. In electrical devices such as HEMTs, if a charge accumulation is expected at a given interface for a given polarity, the occurrence of the opposite polarity will cause instead a depletion, as displayed in Fig. 2.

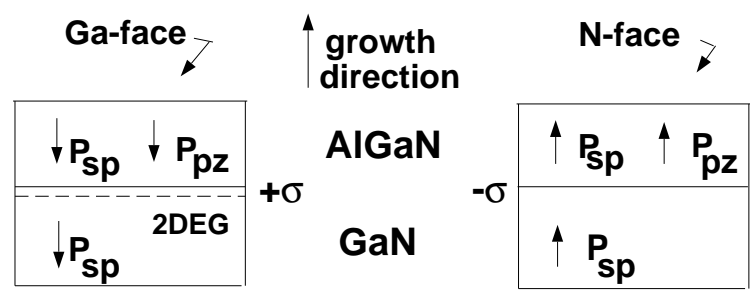

FIG. 2. Interplay of polarity and 2DEG formation in the HEMT channel (adapted from Ref. 22]).

Of course, the magnitude of polarizations in the layers will determine the density of the 2-D gas, hence quan- titative agreement can be expected only including both components. Indeed, in $\mathrm{CV}$ experiments on controlledpolarity HEMT structures [22], the appearance (or nonappearance) of a 2-D gas at a specific interface was shown to correlate 22 with the existence of the spontaneous polarization component, and its value was estimated in fair agreement with our calculations [4].

10 - Piezoelectric and spontaneous polarization can be independently controlled to a large extent, basically because only the first depends on strain. It is easy to show 13 that it is possible to obtain completely unstrained, yet polarized layers, as well as strained yet unpolarized systems. In the first case, the piezo component vanishes by construction, hence spontaneous polarization is unambiguously the only remaining source of field, and can therefore be measured. In the second case, the composition must be such that polarization differences are zero across all interfaces, whence the fields vanish in all layers. It is rather clear that, since realizing this situation requires compensating piezo and spontaneous components, alloying with $\mathrm{Al}$ and In will be needed. For instance, the field-free case should be obtained with GaN-matched $\mathrm{GaN} / \mathrm{Al}_{0.10} \mathrm{In}_{0.06} \mathrm{Ga}_{0.84} \mathrm{~N}$ MQWs with equally thick wells and barriers. Also GaNmatched $\mathrm{GaN} / \mathrm{Al}_{0.68} \mathrm{In}_{0.32} \mathrm{~N}$ MQWs should exhibit the same behavior. In practice, one would measure the red shift vs. thickness for a series of GaN/AlInGaN MQWs starting at null In content; upon progressively increasing the In content, the shift should be first seen to vanish, and then to reappear for larger In concentration. Problems (or opportunities) that should be watched out for in modeling are that the interface band offset may switch to type-II, and that alloy phase separation is likely. Of course, the "critical" concentrations mentioned above must be taken as indicative only, in view of their sensitivity to the intricate interdependencies of strain, lattice parameters, piezoconstants, and spontaneous polarization values.

We thank Oliver Ambacher for discussions and for pointing out recent experimental work in the field. VF thanks the Alexander von Humboldt Foundation for support of his stays at the WSI. Support by INFM under a Section-E PAISS project is acknowledged.

[1] See the recent review by O. Ambacher, J. Phys. D: Appl. Phys. 31, 2653 (1998).

[2] R.D. King-Smith and D. Vanderbilt, Phys. Rev. B 47, 1651 (1993); R. Resta, Rev. Mod. Phys. 66, 899 (1994)

[3] D. Vanderbilt and R.D. King-Smith, Phys. Rev. B 48, 4442 (1993).

[4] F. Bernardini, V. Fiorentini, and D. Vanderbilt, Phys. Rev. B 56, R10024 (1997). 
[5] F. Bernardini, V. Fiorentini, and D. Vanderbilt, Phys. Rev. Lett. 79, 3958 (1997).

[6] F. Bernardini and V. Fiorentini, Phys. Rev. B 57, R9427 (1998).

[7] F. Bernardini and V. Fiorentini, Phys. Rev. B 58, 15292 (1998).

[8] F. Bernardini and V. Fiorentini, physica status solidi, in print.

[9] Polarization in the nitride alloys used in heterostructures is estimated by Vegard-like linear interpolation between binary compound data as

$$
\begin{aligned}
\mathbf{P}_{\mathrm{T}}\left(\mathrm{Al}_{\mathrm{x}} \mathrm{Ga}_{1-\mathrm{x}} \mathrm{N}\right) & \simeq x \mathbf{P}_{\mathrm{AlN}}^{(0)}+(1-x) \mathbf{P}_{\mathrm{GaN}}^{(0)} \\
& +\left[x \overleftrightarrow{e}_{\mathrm{AlN}}+(1-x) \overleftrightarrow{e}_{\mathrm{GaN}}\right] \vec{\epsilon}(x)
\end{aligned}
$$

for e.g. AlGaN, where the spontaneous polarization vec-

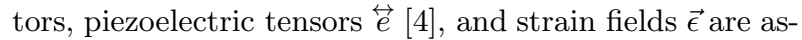
sumed to be known. Similar relations hold for quaternary solutions. While there is no guarantee that this Vegardlike estimate be accurate, there is no alternative to this route at this point in time.

[10] G. Burns, Solid State Physics (Academic Press, San Diego 1990), Sec 5.4.

[11] K. Shimada, T. Sota, K. Suzuki, and H. Okumura, Jap. J. Appl. Phys. 37, L1421 (1998).

[12] F. Della Sala, A. Di Carlo, P. Lugli, F. Bernardini, V. Fiorentini, R. Scholz, and J.-M. Jancu, Appl. Phys. Lett. 74, 2002 (1999).

[13] V. Fiorentini, F. Bernardini, F. Della Sala, A. Di Carlo, and P. Lugli, Phys. Rev B. 60, in print (1999).

[14] Not related to dangling bonds as in abrupt heterovalent junctions, and not implying interface instability [6].

[15] T. Takeuchi, S. Sota, M. Katsuragawa, M. Komori, H. Takeuchi, H. Amano, and I. Akasaki, Jpn. J. Appl. Phys.
36, L382 (1997); S. F. Chichibu, A. C. Abare, M. S. Minsky, S. Keller, S. B. Fleischer, J. E. Bowers, E. Hu, U. K. Mishra, L. A. Coldren, S. P. DenBaars, amd T. Sota, Appl. Phys. Lett. 73, 2006 (1998).

[16] J. S. Im, H. Kollmer, J. Off, A. Sohmer, F. Scholz, and A. Hangleiter, Phys. Rev. B 57, R9435 (1998); B. Gil, P. Lefebvre, J. Allegre, H, Mathieu, N. Grandjean, M. Leroux, J. Massies, P. Bigenwald, and P. Christol, Phys. Rev. 59, 10246 (1999); P. Lefebvre, J. Allegre, B. Gil, H, Mathieu, N. Grandjean, M. Leroux, J. Massies, and P. Bigenwald, Phys. Rev. 59, 15363 (1999).

[17] R. Langer, J. Simon, V. Ortiz, N. T. Pelekanos, A. Barski, R. André, and M. Godlewski, Appl. Phys. Lett. 74, 3827 (1999).

[18] L.-H. Peng, C.-W. Huang, and L.-H. Lou, Appl. Phys. Lett. 74, 795 (1999).

[19] T. Deguchi, A. Shikanai, K. Torii, T. Sota, S. Chichibu, and S. Nakamura, Appl. Phys. Lett. 72, 3329 (1998).

[20] E. T. Yu, X. Z. Dang, L. S. Yu, D. Qiao, P. M. Asbeck, S. S. Lau, G. J. Sullivan, K. S. Boutros, and J. M Redwing, Appl. Phys. Lett. 73, 1880 (1998)

[21] A. D. Bykhovski R. Gaska, and M. S. Shur, Appl. Phys. Lett. 73, 3577 (1998);

[22] O. Ambacher, J.Smart, J. R. Shealy, N. G. Weimann, K. Chu, M. Murphy, W. J. Schaff, L. F. Eastman, R. Dimitrov, L. Wittmer, M. Stutzmann, W. Rieger, and J. Hilsenbeck, J. Appl. Phys. 85, 3222 (1999).

[23] R. Oberhuber, G. Zandler, and P. Vogl, Appl. Phys. Lett. 73, 818 (1998).

[24] P. Kodozoy, M. Hansen, S. P. DenBaars, and U. K. Mishra, Appl. Phys. Lett. 74, 3681 (1999). 